\title{
ECLETICA
}

www.scielo.br/eq

Volume 30, número 2, 2005

\section{Remoção de compostos tóxicos de solução aquosa por adsorção com zeólita sintetizada a partir de cinzas de carvão}

\author{
D. A. Fungaro, J. C. Izidoro, R. S. Almeida \\ Centro de Química e Meio Ambiente - Instituto de Pesquisas Energéticas e Nucleares \\ CEP 05422-970 - São Paulo SP - Brasil
}

\begin{abstract}
Resumo: A capacidade das zeólitas sintetizadas a partir da cinza de carvão brasileiro na remoção dos íons de $\mathrm{Cd}, \mathrm{Zn}$ e do azul de metileno de soluções aquosas foi investigada. O material zeolítico preparado com a cinza de carvão do filtro manga apresentou a eficiência de remoção mais alta para os poluentes. A seletividade aos íons metálicos deste produto foi determinada como: $\mathrm{Cd}^{2+}>\mathrm{Zn}^{2+}$. As capacidades máximas de adsorção encontradas foram $36,1 \mathrm{mg} \mathrm{g}^{-1}$ para o $\mathrm{Zn}^{2+}, 76,3 \mathrm{mg} \mathrm{g}^{-1}$ para o $\mathrm{Cd}^{2+} \mathrm{e} 3,93 \mathrm{mg} \mathrm{g}^{-1}$ para o corante.
\end{abstract}

Palavras-chave: material zeolítico; adsorção; cinza leve de carvão.

\section{Introdução}

Um dos resíduos sólidos mais significativos em termos de volume no Brasil são as cinzas de carvão geradas em usinas termelétricas. A necessidade de retirar as cinzas das usinas a baixo custo faz com que se adotem práticas de disposição em áreas impróprias e sem medidas de proteção adequadas. A lixiviação das cinzas causa a contaminação do solo, das águas subterrâneas e superficiais por substâncias tóxicas.

As principais aplicações das cinzas são na fabricação e incorporação ao cimento e uso como material fertilizante, mas isto ainda ocorre em pequena escala ( $30 \%)$. Uma das maneiras de reduzir os impactos ambientais decorrentes da disposição destes resíduos no meio ambiente consiste na ampliação das potencialidades de sua utilização. Uma alternativa de aproveitamento destes resíduos é a transformação das cinzas de carvão em um adsorvedor de baixo custo capaz de remover substâncias tóxicas de águas contaminadas.

As cinzas de carvão mineral são constituídas basicamente de sílica e alumina sendo possível convertê-las em material zeolítico após tratamento hidrotérmico com hidróxido de sódio. Os metais tóxicos que a cinza contém são removidos na solução básica que é encaminhada para tratamento posterior. Os métodos de conversão direta combinam a liberação de $\mathrm{Si}$ e $\mathrm{Al}$ e a cristalização de zeólitas em um simples estágio resultando, geralmente, em um produto final que contém 20 - 75\% de zeólita dependendo das condições do processo.

A literatura apresenta inúmeros trabalhos sobre a conversão de cinzas em zeólita por tratamento hidrotérmico alcalino e a sua utilização na remoção de metais em água [1-10], mas há poucos estudos sobre esta alternativa empregando-se cinzas de carvão geradas no Brasil [11-12] e em relação à remoção de compostos orgânicos [13]. O carvão brasileiro apresenta características próprias que irão influenciar as propriedades das cinzas e das suas respectivas zeólitas e, conseqüentemente, a capacidade de troca iônica do adsorvedor.

Os diferentes tipos de cinzas de carvão amostradas na termelétrica irão gerar zeólitas com composições variadas durante o tratamento hidrotérmico. A natureza da zeólita influi no processo de adsorção dos compostos. O objetivo do trabalho foi determinar a eficiência das zeólitas preparadas com 
cinzas leves de carvão em remover íons metálicos e compostos corantes de soluções aquosas.

\section{Material e métodos}

\section{Material}

Todos os reagentes usados eram de grau analítico (Merck). As soluções dos íons metálicos e do azul de metileno foram preparadas a partir da diluição de solução estoque com água ultrapura do sistema Millipore Milli-Q. Agitador mecânico com temperatura controlada, centrífuga e espectrofotômetro Cary 1E - Varian foram utilizados.

\section{Preparação das zeólitas}

As cinzas de carvão da Usina Termelétrica de Figueira, localizada no Paraná, foram utilizadas no estudo. As zeólitas foram preparadas a partir dos seguintes tipos de cinzas leves: retidas no filtro manga (ZM) e retidas no filtro ciclone (ZC). O procedimento de Henmi [14] foi seguido para o tratamento hidrotérmico: a amostra contendo $30 \mathrm{~g}$ de cinzas de carvão foi colocada com $240 \mathrm{~mL}$ de $\mathrm{NaOH}$ $3,5 \mathrm{~mol} \mathrm{~L}^{-1}$ e aquecida em estufa, à $100^{\circ} \mathrm{C}$, por 24 horas. A suspensão foi filtrada e o sólido foi repetidamente lavado com água deionizada e seco em estufa a $40^{\circ} \mathrm{C}$. As zeólitas foram analisadas por fluorescência de raios-X (XRFS RIX 3000 - Rigaku) e por difração de raios-X (Bruker-AXS D8). A capacidade de troca catiônica (CTC) dos materiais zeolíticos foi obtida usando solução de acetato de sódio e de amônio [8].

\section{Estudos sobre a remoção dos compostos tóxicos}

A remoção dos compostos pelas zeólitas foi realizada por processos descontínuos. Uma alíquota de solução do composto $(100 \mathrm{~mL})$ foi misturada com $1 \mathrm{~g}$ de zeólita. O tempo de equilíbrio das isotermas foi determinado em $24 \mathrm{~h}$ para os íons metálicos e $2 \mathrm{~h}$ para o azul de metileno a partir de estudos cinéticos.O sobrenadante foi separado por centrifugação e a concentração final nesta solução foi determinada. Os íons metálicos $\mathrm{Zn}^{2+}$ e $\mathrm{Cd}^{2+}$ foram determinados por titulação complexiométria com EDTA. A concentração dos metais estava na faixa de 261 a $895 \mathrm{mg} \mathrm{L}^{-1}$. A concentração do corante azul de metileno foi determinada por espectrofotometria em $\ddot{e}_{\max }=650 \mathrm{~nm}$ após ajuste do $\mathrm{pH}$ ótimo $(\mathrm{pH}=5)$, na faixa de trabalho de $1,6 \mathrm{a}$ $32 \mathrm{mg} \mathrm{L}^{-1}$.

\section{Resultados e Discussão}

As zeólitas ZM e ZC apresentaram conteúdos de alumina e sílica muito similares $\left(\mathrm{SiO}_{2}+\mathrm{Al}_{2} \mathrm{O}_{3}\right.$ $65-70 \%$ ), conteúdo relativamente alto de óxido férrico $(10-16 \%)$ e óxido de sódio $(7-16 \%)$ e razão $\mathrm{SiO}_{2} / \mathrm{Al}_{2} \mathrm{O}_{3} 1,65$ e 1,90, respectivamente. Encontraram-se os óxidos de cálcio, potássio, titânio, enxofre, magnésio e outros compostos em quantidades menores que 5\%. A análise por DR-X revelou que o principal produto obtido após o tratamento hidrotérmico era a zeólita $\mathrm{NaP} 1$ com traços de quartzo, mulita e hidroxisodalita. Os valores de CTC encontrados foram 176,9 meq $100 \mathrm{~g}^{-1}$ para a ZM e 138,1 meq $100 \mathrm{~g}^{-1}$ para ZC. A comparação dos valores da capacidade de troca catiônica da zeólita sintética comercial $\mathrm{NaP} 1$ com aqueles encontrados com as zeólitas ZM e ZC indicou que o conteúdo de material zeolítico obtido na síntese hidrotérmica foi de 35,4 e 27,6\%, respectivamente. A área superficial específica (procedimento padrão BET adsorção de $\mathrm{N}_{2}$ ) dos materiais zeolíticos apresentou valores entre $30-34 \mathrm{~m}^{2} \mathrm{~g}^{-1}$ similar aos dos materiais argilosos e caulinitas [15-16].

Os dados de adsorção foram ajustados pelos modelos de Langmuir e Freundlich que são os mais freqüentemente usados para descrever isotermas para aplicações em tratamento de águas e efluentes [17-18]. As isotermas de adsorção dos íons metálicos e do azul de metileno estão mostradas nas Figuras 1 e 2, respectivamente.

As constantes de Langmuir e Freundlich foram determinadas por regressão linear das equações linearizadas e estão listados na Tabela 1 , juntamente com os dados da literatura de outros adsorvedores.

As isotermas do $\mathrm{Zn}^{2+} \mathrm{e} \mathrm{Cd}{ }^{2+}$ podem ser classificadas conforme o Tipo L2 [22]. Os valores dos coeficientes de correlação das retas $\left(\mathrm{r}^{2} \mathrm{e}\right.$ " 0,99$)$ mostraram que ambos os modelos de isotermas de Langmuir e Freundlich podem adequadamente descrever os dados de adsorção. A ordem de seletividade dos cátions pelas zeólitas $\mathrm{ZM} \mathrm{e} \mathrm{ZC} \mathrm{foi} \mathrm{Cd}^{2+}>\mathrm{Zn}^{2+}$. A afinidade de uma zeólita está relacionada com muitos fatores [23] e no caso, reflete o decréscimo do raio dos íons metálicos hidratados e a energia de hidratação. As zeólitas ZM e ZC alcançaram capacidades de adsorção dos íons metálicos (35 a 76 mg g$\left.{ }^{1}\right)$ maiores do que aquelas previamente relatadas em literatura [7] e eficiência de retenção e" $70 \%$. 


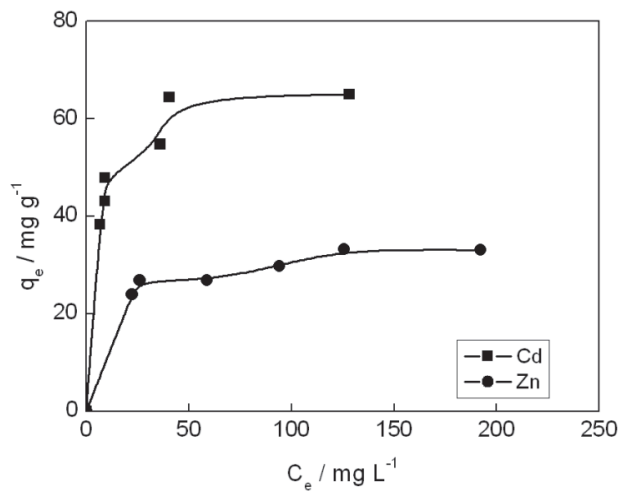

(a)

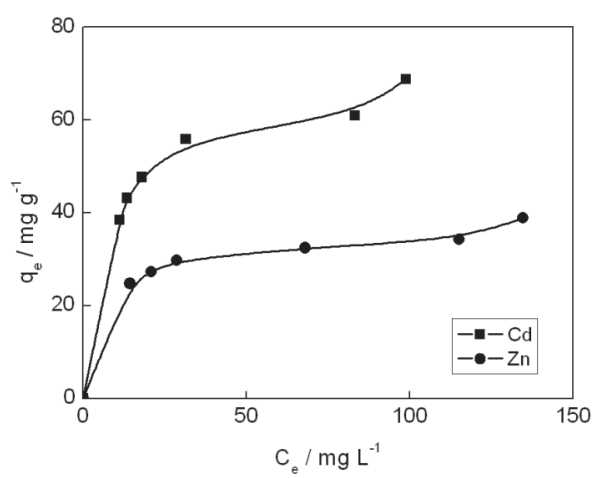

(b)

Figura 1. Isotermas de adsorção para o $\mathrm{Zn}^{2+}$ e o $\mathrm{Cd}^{2+}$ com: (a) zeólita ZM; (b) zeólita ZC.

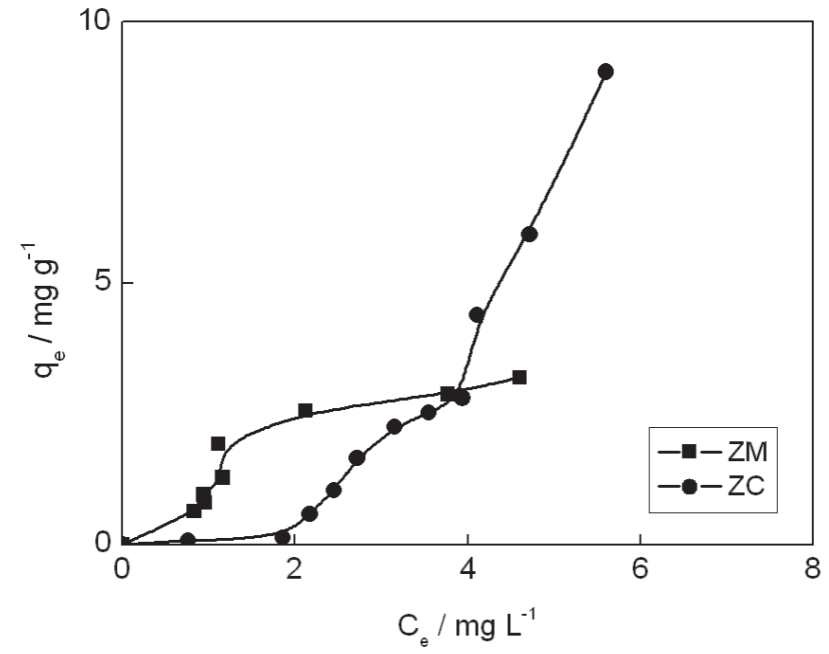

Figura 2. Isotermas de adsorção das zeólitas ZM e ZC para o azul de metileno.

A isoterma de equilíbrio para o azul de metileno com ZM mostrou um comportamento concordante com o Tipo L2 [22], com os dados se ajustando aos modelos de Langmuir e Freundlich ( $\mathrm{r}^{2} \mathrm{e}$ " 0,99). AZM exibiu um valor de capacidade de adsorção máxima 3,5 vezes maior do que aquele obtido com o uso de cinza de carvão como adsorvedor [13]. Já a isoterma com ZC apresentou uma curva na forma sigmoidal indicando que o equilíbrio foi favorável até o valor de concentração de equilíbrio $\left(\mathrm{C}_{\mathrm{e}}\right)$ de $\sim 4 \mathrm{mg} \mathrm{L}^{-1} \mathrm{e}$, depois tornou-se desfavorável mostrando uma curva ascendente. Este comportamento corresponde á isoterma Tipo L3 indicando a formação rápida de uma segunda ca- mada de adsorção [22]. Os dados não se ajustaram aos modelos de adsorção de Langmuir e Freundlich. Uma eficiência de retenção do azul de metileno e" $88 \%$ foi obtida com ambos adsorvedores.

O processo de adsorção foi favorável, conforme indicado pelas constantes $\quad \mathrm{R}_{\mathrm{L}}\left(0<\mathrm{R}_{\mathrm{L}}\right.$ $<1)$ e n $(2<\mathrm{n}<10)$ na faixa de concentração estudada [24-25]. A zeólita ZM apresentou melhor eficiência de adsorção do que ZC devido principalmente aos seguintes fatores: (1) a menor relação $\mathrm{SiO}_{2} / \mathrm{Al}_{2} \mathrm{O}_{3}$ na composição química da zeólita e conseqüente, maior capacidade de troca catiônica; (2) o maior conteúdo de $\mathrm{NaP} 1$ formado na cinza modificada. 
Tabela 1. Parâmetros dos modelos de isoterma de Freundlich e Langmuir para as zeólitas ZM e ZC e diferentes adsorvedores.

\begin{tabular}{cccccccc}
\hline Adsorvedor & $\begin{array}{c}\text { Compostos } \\
\text { tóxicos }\end{array}$ & \multicolumn{2}{c}{ Freundlich } & & Langmuir & & Ref. \\
\hline $\mathrm{ZM}$ & $\mathrm{Kn}$ & 17,4 & 6,89 & 36,1 & 0,15 & 0,012 & $\begin{array}{c}\text { presente } \\
\text { trabalho } \\
\text { presente } \\
\text { trabalho }\end{array}$ \\
& $\mathrm{Cd}$ & 24,7 & 4,40 & 76,3 & 0,092 & 0,014 & \\
& $\mathrm{AM}$ & 1,89 & 2,93 & 3,93 & 0,85 & 0,036 & $\begin{array}{c}\text { presente } \\
\text { trabalho }\end{array}$ \\
& & & & & & & \\
ZC & $\mathrm{Zn}$ & 14,9 & 6,64 & 35,1 & 0,082 & 0,023 & $\begin{array}{c}\text { presente } \\
\text { trabalho } \\
\text { presente } \\
\text { trabalho }\end{array}$ \\
& $\mathrm{Cd}$ & 30,6 & 6,39 & 67,4 & 0,21 & 0,0060 & \\
zeólitas & $\mathrm{Zn}$ & - & - & $2,7-5,5$ & - & - & 19 \\
naturais & $\mathrm{Cd}$ & - & - & $3,7-6,7$ & - & - & 19 \\
cinzas de & $\mathrm{Zn}$ & - & - & 0,301 & 0,178 & 0,15 & 20 \\
carvão & $\mathrm{Cd}$ & - & - & 0,0345 & 3,80 & 0,040 & 21 \\
& $\mathrm{AM}$ & - & - & 1,11 & 0,04 & - & 13 \\
argila & $\mathrm{AM}$ & - & - & $4,4-6,3$ & $0,06-0,78$ & - & 16 \\
\hline
\end{tabular}

$\mathrm{ZM}$ = zeólita preparada com cinza do filtro manga; $\mathrm{ZC}=$ zeólita preparada com cinza do filtro ciclone; $\quad \mathrm{AM}=$ azul de metileno. (*) $\left(\mathrm{mg} \mathrm{g}^{-1}\right)\left(\mathrm{L} \mathrm{mg}^{-1}\right)^{1 / n}$

\section{Conclusão}

A zeólita sintética preparada com cinzas de carvão mostrou-se eficiente como material alternativo para ser usado no tratamento de efluentes com altos níveis de zinco e cádmio (indústrias do setor metal-mecânico) ou com substâncias corantes (indústrias têxteis). A zeólita sintetizada a partir da cinza leve do filtro manga apresentou a melhor capacidade de adsorção dos compostos tóxicos. A abundância do resíduo e o baixo custo viabilizam a adoção do processo na gestão ambiental de usinas termelétricas.

\section{Agradecimentos}

Os autores agradecem o Conselho Nacional de Desenvolvimento Científico e Tecnológico CNPq e a Carbonífera do Cambuí Ltda.

Recebido em: 29/11/2004

Aceito em: 22/02/2005 
D. A. Fungaro, J. C. Izidoro, R. S. Almeida. Removal of toxics compounds from aqueous solution by adsorption with zeolite synthesized from coal ashes

Abstract: The capacity of synthesized zeolites from Brazilian coal ash for the removal of Cd, Zn ions and methylene blue from aqueous solutions has been investigated. The zeolitic material prepared with coal ash from baghouse filter showed the highest efficiency for pollutants removal. The metal ion selectivity of this product was determined as: $\mathrm{Cd}^{2+}>\mathrm{Zn}^{2+}$. The maximum sorption capacities found were $36.1 \mathrm{mg} \mathrm{g}^{-1}$ for $\mathrm{Zn}^{2+}$, $76.3 \mathrm{mg} \mathrm{g}^{-1}$ for $\mathrm{Cd}^{2+}$ and $3.93 \mathrm{mg} \mathrm{g}^{-1}$ for dye.

Keywords: zeolitic material; adsorption; coal fly ash.

\section{Referências}

[1] C. F. Lin, H.C. His, Environ. Sci. Technol. 29 (1995) 1109.

[2] A. Singer, V. Berkgaut, Environ. Sci. Technol. 29 (1995) 1748 .

[3] C. Amrhein, G. H. Haghnia, T. S. Kim, P. A. Mosher, R. C. Gagajena, T. Amanios, L. Torre, Environ. Sci. Technol. 30 (1996) 735.

[4] W-H. Shih, H-L. Chang, Mater. Lett. 28 (1996) 263.

[5] G. Steenbruggen, G. G. Hollman, J. Geochem. Explor. 62 (1998) 305.

[6] M-G. Lee, G. Yi, B-J. Ahn, F. Roddick, Korean J. Chem. Eng. 17 (2000) 325.

[7] X. Querol, F. Plana, A. Alastuey, A. Lopez-Soler, A. Medinaceli, A. Valero, M.J. Domingo, E. Garcia-Rojo, Fuel 80 (2001) 857.

[8] J. Scott, D. Guang, K. Naeramitmarnsuk, M. Thabuot, J. Chem. Technol. Biotechnol. 77 (2002) 63.

[9] R. Juan, S. Hernandez, X. Querol, J. M. Andrés, N. Moreno, J. Chem. Technol. Biotechnol. 77 (2002) 299.

[10] X. Querol, N. Moreno, J. C. Umaña, A. Alastuey, E. Hernández, A. López-Soler, F. Plana, Int. J. Coal Geol. 50 (2002) 413.

[11] D. A. Fungaro, M. G. Silva, Quim. Nova 25 (2002) 1081.
[12] D. A. Fungaro, M. S-M. Flues, A. P. Celebroni, Quim. Nova 27 (2004) 582.

[13] C. D. Woolard, C. R. Strong, C. R. Erasmus, Appl. Geochem. 17 (2002) 1159.

[14] T. Henmi, Clay Sci. 6 (1987) 277.

[15] M. R. M. P. Aguiar, A. C. Novaes, Quim. Nova 25 (2002) 1154.

[16] A. Gurses, S. Karaca, C. Dogar, R. Bayrak, M. Açikyildiz, M. Yalçin, J. Colloid Interface Sci. 269 (2004) 310.

[17] S. D. Faust, O. M. Aly, Adsorption Process for Water Treatment, Butterworths, London, 1987.

[18] J. R. Perrich, Actived Carbon Adsorption for Waste Water Treatment, CRC Press, Boca Raton, 1981.

[19] S. K. Ouki, M. Kavannagh, Wat. Sci. Tech. 39 (1999) 115.

[20] B. Bayat, J. Hazard. Mat. B95 (2002) 251.

[21] B. Bayat, J. Hazard. Mat. B95 (2002) 275.

[22] C. H. Giles, D. Smith, J. Colloid Interface Sci. 47 (1974) 755 .

[23] A. Dyer, Zeolite surfaces and reactivity. In: D. J. Vaughan, R. D. A. Patrick, Eds, Mineral Surfaces. Chapman and Hall, London, 1995, p.333.

[24] K. R. Hall, L. C. Eagleton, A. Acrivos, T. Vermeulen, Ind. Eng. Chem Fundam. 5 (1966) 212.

[25] W. A. Helby, Chem. Eng. 59 (1952) 153 